\title{
Tumores neuroendocrinos de origen pulmonar. Reporte de un caso
}

\author{
JOSEFINA CAVIEDES M.*, CRISTÓBAL APARICIO S.*, ANA CUADRA R.*, \\ JOSÉ VELÁSQUEZ M.** y ALICIA BENAVIDES M.****
}

\section{Neuroendocrine tumors of lung's origin, a case report}

Introduction or case story: Young female patient (24 years-old), without known morbid precedents. She comes for a ten-days period of symptoms characterized by fever of up to $38.5^{\circ} \mathrm{C}$ and a dyspnoea grade III. Physical exam showed decreased vesicular murmur on the right pulmonary base with dullness and positive vocal vibrations. Exams: Thorax X-ray: Atelectasis condensation on the right pulmonary base. CT chest scan without contrast: Nodule located in an intermediate bronchus which generates atelectasis in the basal bronchi. Fibro-bronchoscopy (FOB): A tumour-like injury blocking 100\% of the right intermediate bronchus' duct. Biopsy: Pulmonary tissue with haemorrhagic areas, granular tissue with small cellular clusters of lobular disposal and glandular shape with eccentric central nuclei cells, with homogenous chromatin and without atypical mitosis. Immunohistochemistry: Intensely positive cells to synaptophysin and CD56. Diagnosis: Neuroendocrine Typical Carcinoid Tumor. Comments: The patient evolves without progression of dyspnoea, she is waiting for a surgical resolution of tumour at National Institute of Thorax.

Key words: Neuroendocrine tumors; Carcinoid tumor; Pulmonary atelectasis; Immunohistochemistry; Synaptophysin.

\section{Resumen}

Introducción o historia del caso: Mujer joven de 24 años de edad, sin antecedentes mórbidos, acudió por cuadro de 10 días de evolución, de fiebre de hasta $38,5^{\circ} \mathrm{C}$ y disnea grado III. Al examen fisico destacó a nivel pulmonar: murmullo pulmonar disminuido en base pulmonar derecha, matidez de la misma zona y vibraciones vocales positivas. Exámenes: Radiografia de Tórax: Condensación atelectásica en base pulmonar derecha. TAC de Tórax sin contraste: Imagen nodular a nivel de bronquio intermedio, que genera atelectasia en bronquios basales. Fibrobroncoscopía (FBC): Lesión tumoral que ocluye el $100 \%$ del lumen para bronquio intermedio derecho. Biopsia: Tejido pulmonar con áreas de hemorragia, tejido granulatorio y tumor con acúmulos celulares de disposición lobular y glanduliforme, con núcleos centrales excéntricos, cromatina homogénea, sin atipias. Inmunohistoquímica: Células intensamente positivas para sinaptofisina, y CD-56. Diagnóstico: Tumor Neuroendocrino Carcinoide típico Comentarios: Paciente evoluciona sin progresión de su disnea, esperando resolución quirúrgica del tumor en Instituto Nacional del Tórax.

Palabras clave: Tumores neuroendocrinos; Tumor Carcinoide; Atelectasia pulmonar; Inmunohistoquímica; Sinaptofisina.

\footnotetext{
* Internos de Medicina $6^{\circ}$ año, Universidad San Sebastián, Santiago.

** Medicina Interna, Broncopulmonar Clínica Hospital del Profesor, Docente Universidad San Sebastián.

***Departamento de Anatomopatología, Clínica Hospital del Profesor, Santiago.
} 


\section{Introducción}

El tejido pulmonar de forma normal contiene en el interior de su epitelio, células inmersas que tienen un origen neuroendocrino, estas células tienen una actividad secretora, y poseen la capacidad de consumir y descarboxilar los precursores de aminas, éstas son las llamadas células de Kulchitzky, o células enterocromafines ${ }^{1}$. El origen primitivo de esta estirpe celular, le provee la característica de ser pluripotenciales, y que además tienen la capacidad de producir neurotransmisores, sin la necesidad de tener axones, ni de hacer sinapsis ${ }^{2}$. Es por ello, que las células neuroendocrinas, son las células que, durante la formación pulmonar, ya están en el epitelio primitivo, aumentando en número en el período prenatal más tardío, y llegando a un peak en el período neonatal, persistiendo posteriormente como una población activa en el epitelio pulmonar ${ }^{3}$.

Son estas células, cuya proliferación descontrolada da origen a los tumores neuroendocrinos (TNE) de origen pulmonar. Con el avance de la biología celular y molecular se ha descubierto que puede haber otros orígenes. Según la procedencia embrionaria estos pueden estar en otros tejidos, como el gastrointestinal. Siendo incluso más frecuente en estos sitios con un 70\% del total de los TNE, siendo sólo un 25\% los de origen pulmonar $^{4,5}$. Si bien las neoplasias neuroendocrinas en general tienden a compartir características histológicas símilares, hay aspectos clínicos y patológicos que las diferencian dependiendo del órgano de origen ${ }^{4}$.

Según la clasificación de la OMS del año 2004, los TNE de origen pulmonar se distinguen 4 subtipos de tumores, los cuales están divididos según histología y según grado de malignidad: Bajo grado (Carcinoides típicos), Grado intermedio (Carcinoides Atípicos), Alto grado (Carcinoma neuroendocrino de células no pequeñas, y el carcinoma de células pequeñas) ${ }^{1-3}$. Ninguno de ellos puede ser llamado "benigno", ya que, todos tienen la capacidad de metastizar. Sin embargo, dentro de los que tienen menor capacidad metastásica y, por ende, tienen menor grado de malignidad están los carcinomas típicos y atípicos, que, no obstante, vienen a ser los menos frecuentes, ocupando tan sólo un $8 \%$ del total de TNE de origen pulmonar ${ }^{2}$. Luego de esta última clasificación, también ha sido reconocida una entidad que es considerada una lesión pre-neoplásica, que es la Hiperplasia difusa de células neuroendocrinas idiopáticas (HDCNI). Esta sería secundaria a una inflamación y/o fibrosis de las vías aéreas, considerada como la manifestación más temprana de esta entidad neuroendocrina ${ }^{2,3}$.

Dentro de los factores de riesgo, se reconoce que existe una fuerte relación entre el tabaquismo y los TNE de alto grado, sin embargo, no es así con los de grado bajo e intermedio, sobre todo con el carcinoma típico ${ }^{1}$. Este último, es el que más se relaciona con pacientes jóvenes, y suelen tener una ubicación más bien central en un $75 \%$ de los casos, por lo cual, clínicamente se presenta frecuentemente con hemoptisis, sibilancias, neumonía recurrente, y dolor torácico ${ }^{2}$. En general, los TNE tienden a estar relacionados con múltiples síntomas secundarios a la excesiva producción hormonal y de aminas, desencadenando cuadros como el síndrome carcinoide siendo más frecuente en los TNE de origen digestivo ${ }^{4}$. Finalmente pueden presentar la sintomatología secundaria al efecto de masa, o bien, ser asintomáticos como sucede en al menos un $50 \%$ de los casos. Ejemplo de ello es el tumor carcinoide de localización periférica, que suele ser sólo un hallazgo clínico ${ }^{2,4}$.

\section{Descripción del caso}

Mujer joven de 24 años de edad, secretaria, de nacionalidad italiana, sin antecedentes mórbidos previamente conocidos, no fumadora, sólo infecciones respiratorias repetidas en los últimos años. Acudió a Servicio de Urgencia, por cuadro de 10 días de evolución, caracterizado por fiebre de hasta $38,5{ }^{\circ} \mathrm{C}$ y disnea grado III, sin tos, sin historia de angina. Negó exposición laboral o ambiental a algún contaminante o agente infeccioso y sin antecedentes familiares significativos. Relata haber tenido cuadros similares en Italia de forma intermitente, sin mayor repercusión. Refiriendo alergia al paracetamol y una dudosa historia de asma en infancia.

Al examen físico destacó estar vigil, orientada en tiempo y espacio, disnea a esfuerzos moderados, con aparente buen estado general, contextura física meso-mórfica. Signos vitales: Frecuencia cardiaca de 78 latidos por minuto, presión arterial: $120 / 80 \mathrm{mmHg}$, frecuencia respiratoria 16 respiraciones por minuto, Saturación de $\mathrm{O}_{2}: 88 \%$ con aire ambiental. A nivel torácico destacó a nivel cardiaco un ritmo regular en dos tiempos, sin auscultarse soplos. A nivel pulmonar un murmullo pulmonar disminuido en la base derecha, con matidez de la misma zona y vibraciones vocales positivas. Abdomen blando, depresible e indoloro, no se palparon víscero-megalias ni masas, con ruidos hidro-aéreos positivos. Extremidades sin 
edema, sin signos clínicos de trombosis venosa profunda (TVP). Sin otro hallazgo positivo, resto del examen físico dentro de límites normales.

Con el fin de establecer la causa de la disminución de murmullo pulmonar, se solicitó una Radiografía de Tórax anteroposterior (AP) y lateral (L), en la cual destaca en su visión AP una gran zona de condensación atelectásica en base pulmonar derecha, sin engrosamiento de la trama peribronquial, y sin broncograma aéreo. No se observó cardiomegalia ni derrame pleural evidente (Figura 1).

Para continuar con estudio de patología pulmonar, se indicó TAC de tórax sin contraste para mejor visualización de imagen sugerente de condensación. En el cual se observó una imagen nodular a nivel de bronquio intermedio, que ocupaba gran porción de su lumen, generando atelectasia de bronquios basales derechos (Figura 2).

Se planteó el diagnóstico de posible nódulo tumoral que ocluiría gran parte de lóbulo medio pulmonar derecho, por lo cual, se programa realizar estudio con Fibrobroncoscopía para obtención de muestras histológicas de dicha lesión. Este estudio confirmó que, a nivel del árbol bronquial derecho, en bronquio intermedio, había la presencia de una lesión de aspecto tumoral, nodular, que sangraba en forma espontánea y ocluía en un $100 \%$ el lumen para dicho bronquio (Figura 3 ).

Se tomó muestra de cuatro fragmentos tisulares que a microscopia óptica se observaban blanquecinos rojizos de 0,2 a $0,5 \mathrm{~cm}$. El examen histológico señaló que correspondía a tejido pulmonar con áreas de hemorragia, tejido granulatorio y tumoral constituido por pequeños acúmulos celulares de disposición lobular y glanduliforme con células de citoplasma claro y núcleos centrales excéntricos, de cromatina homogénea, sin mitosis atípicas ni necrosis de células tumorales. (Figura 4).

Según el estudio inmunohistoquímico, se analizó la presencia de ciertos receptores que podrían complementar aún más el diagnóstico ya arrojado por la histología. Dicho estudio tuvo como resultado que las células tumorales estudiadas eran intensamente positivas a Sinap-

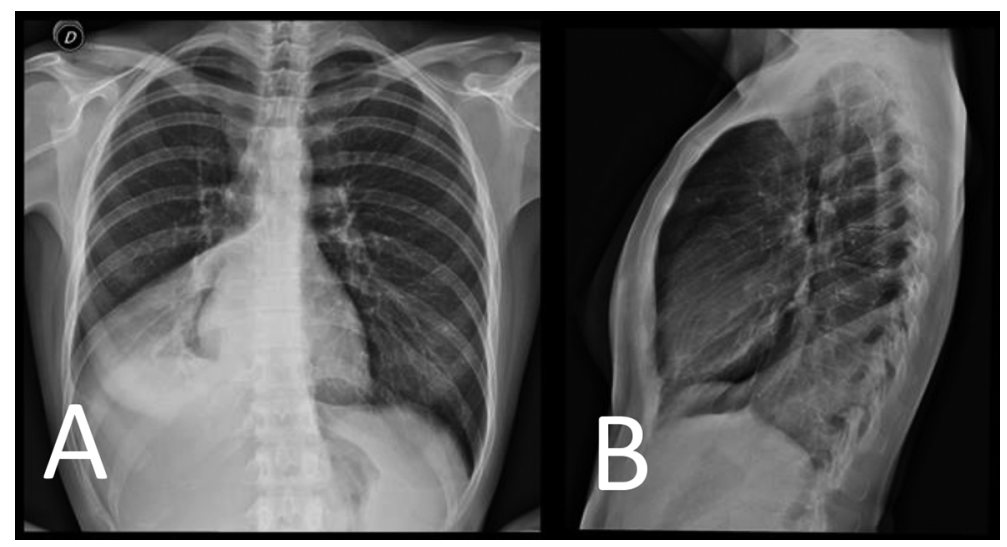

Figura 1. Radiografía de Tórax. Se aprecia condensación atelectásica en base pulmonar derecha. A: Visión anteroposterior (AP). B: Visión lateral (L).

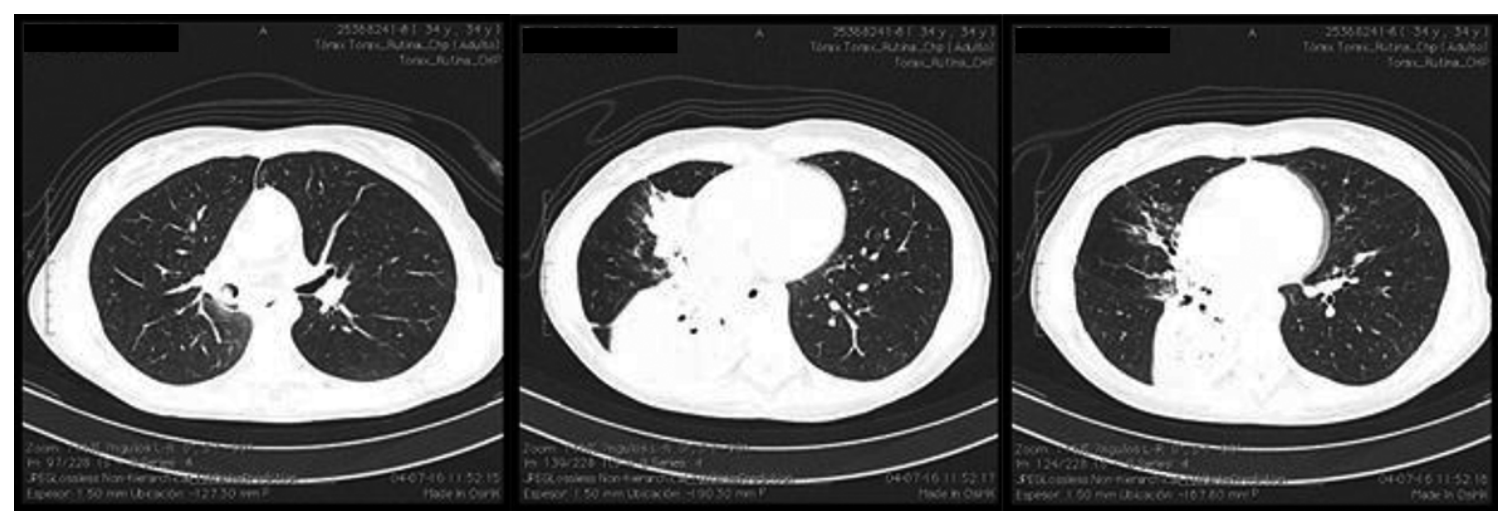

Figura 2. TAC de Tórax sin contraste que muestra imagen nodular. 

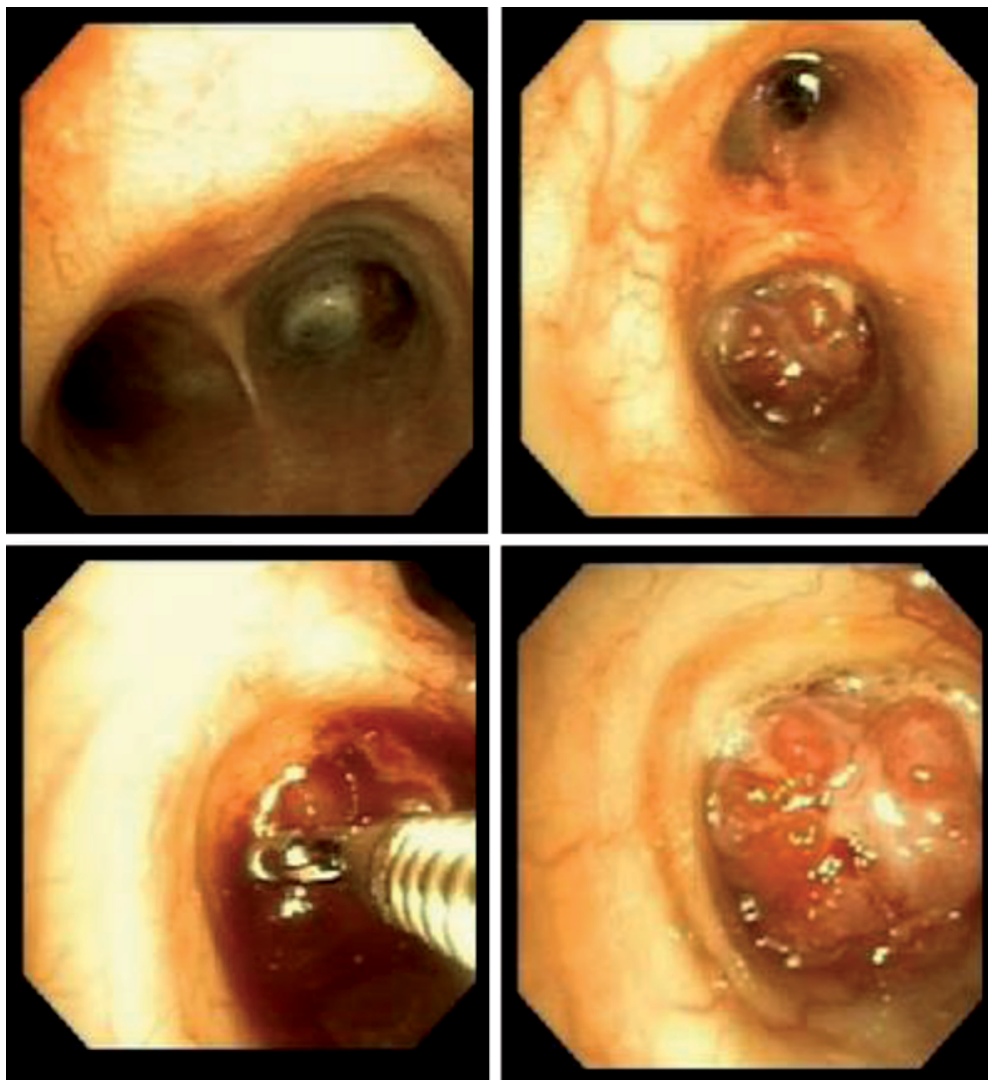

Figura 3. Fibrobroncoscopía que evidencia oclusión luminal de bronquio intermedio derecho.

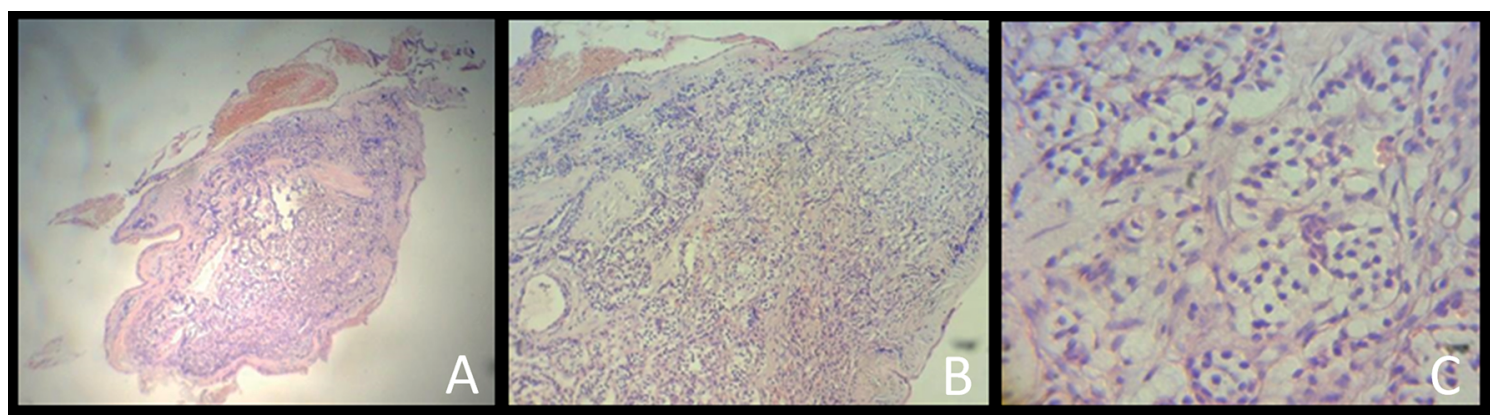

Figura 4. A: Nódulo tumoral, 4x; B: Nidos de células tumorales con núcleos redondeados, 10x; C: Disposición de lóbulos, 40x; Todas las imágenes con Tinción con H-E.

tofisina, y a CD-56, éste último con un patrón de membrana (Figura 5). También se realizó el estudio con HMB-45, sin embargo, fue negativo en las células tumorales. De acuerdo al estudio inmunohistoquímico e histológico, los hallazgos fueron compatibles con un Carcinoma Neuroendocrino de bajo potencial de malignidad, es decir, un Tumor Carcinoide Típico.

Finalmente, dos meses después de haber realizado la primera TAC de Tórax sin contraste, se realizó una Tomografía por emisión de positrones
(PET-SCAN) para completar el estudio, y con el fin de comparar ambos estudios de imágenes y complementarlos. Para la realización del PET-SCAN se utilizó péptidos análogos de los receptores de somatostatina, con el propósito de obtener la captación del tumor carcinoide primario, y observar las posibles metástasis (Figura 6).

Las imágenes evidenciaron un nódulo endobronquial hipercaptante de $20 \mathrm{~mm}$ en relación a bronquio intermedio derecho, que obstruía parcialmente el origen del bronquio lobar inferior 


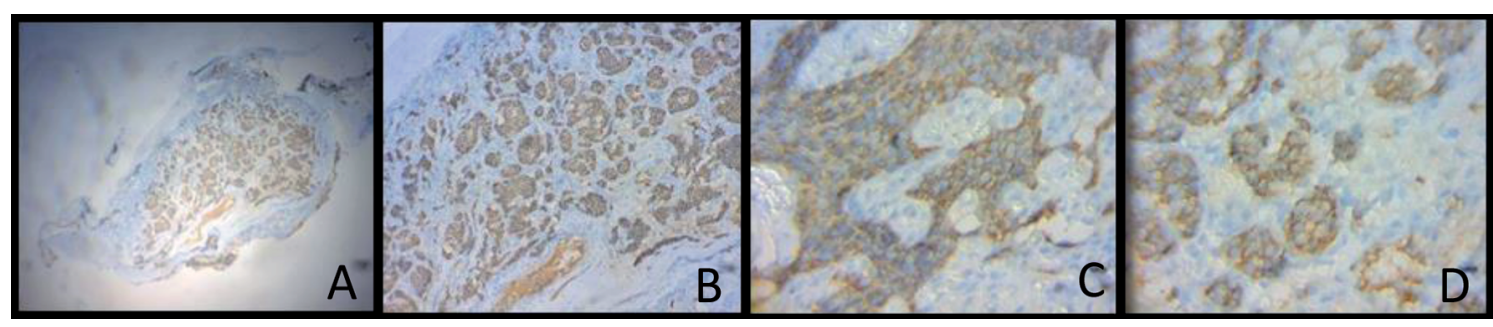

Figura 5: Células teñidas por técnica de inmunohistoquímica A, B y C con Sinaptofisina (4x; 10x; 40x respectivamente) D: con CD-56 (40x).

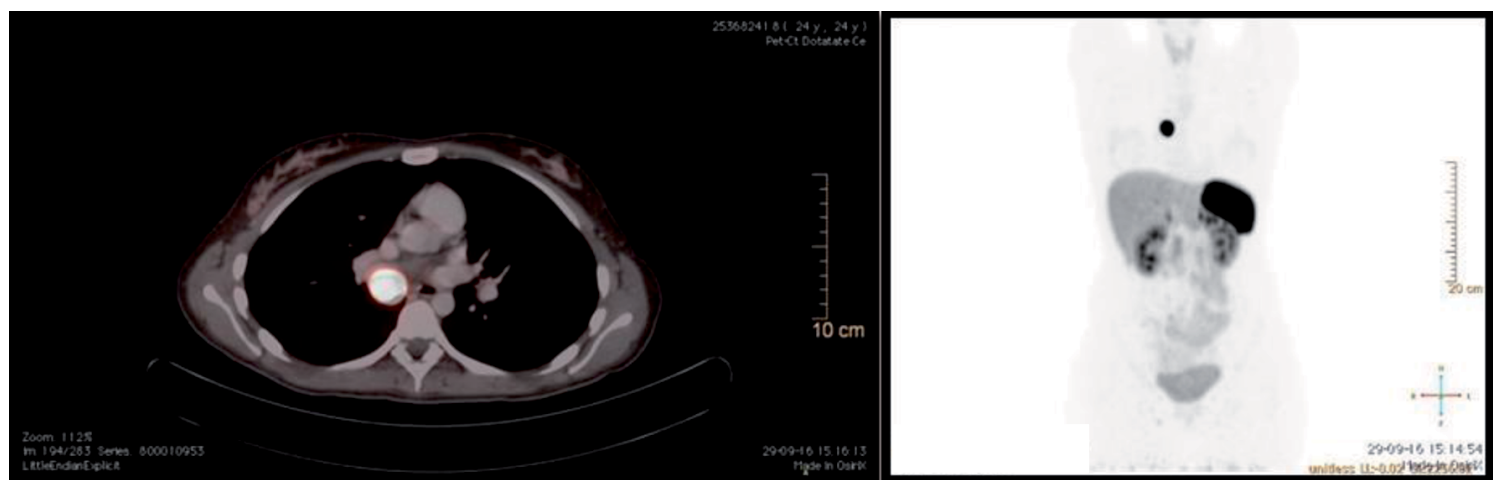

Figura 6. Imágenes obtenidas por Tomografía por emisión de positrones (PET-SCAN) con hipercaptación a nivel de bronquio intermedio derecho.

derecho y para el Lóbulo Medio (LM), sin cambios significativos morfológicos, con SUVmax 111,8 , este sería el Tumor primario conocido. El LM muestra disminución de la densidad y menor cantidad de vasos determinado por fenómenos de atrapamiento aéreo, apreciándose además algunas secreciones endobronquiales a este nivel. El lóbulo inferior derecho se encontró levemente disminuido de volumen también con fenómenos post-obstructivos y algunos tapones mucosos. No se observaron otras lesiones hipercaptantes sugerentes de actividad tumoral maligna.

Confirmado el diagnóstico de tumor carcinoide típico, se programó de inmediato la resolución quirúrgica, con extirpación de tumor en el Instituto Nacional del Tórax, que consistirá en una lobectomía de lóbulo medio e inferior derecho. Hoy la paciente ha evolucionado sin progresión de la disnea, y con manejo médico en cuanto a las infecciones recurrentes de la vía aérea baja, es decir antibiótico terapia y manejo sintomático, además de reposo relativo en su hogar. No ha manifestado ningún tipo de sintomatología nueva, ni complicaciones asociadas al cuadro de hipersecreción neuroendocrina, como síndrome carcinoide, síndrome de Cushing u otro.

\section{Discusión y comentarios}

Se sabe que la presentación clínica más frecuente de los tumores carcinoide típicos está dada principalmente por su ubicación. La cual en su mayoría es igual que en nuestra paciente, de ubicación central. Sin embargo, la sintomatología más comúnmente vista no se presentó en nuestro caso, ya que en ella sólo existieron neumonías repetidas que no produjeron mayor repercusión en su calidad de vida, hasta este último episodio en donde la disnea progresó hacia medianos esfuerzos, impulsándola a consultar para un estudio más acabado.

El punto de partida para la sospecha diagnóstica fue la clínica, y el hallazgo de una imagen anómala en la radiografía de tórax. Para mejor definición de la masa, se realizó una TAC de tórax sin contraste, que evidenció la presencia de una ocupación del bronquio intermedio y bronquio lobular medio. Estudios retrospectivos han determinado que efectivamente la localización más común de los tumores carcinoides es el lóbulo medio derecho, además del lóbulo superior izquierdo ${ }^{1}$. Eventualmente, si el estudio hubiera sido contrastado es probable que la visualización 
de un tumor carcinoide típico hubiera sido más precisa. En general, estos suelen tener una alta vascularización, mejorando notablemente su distinción de una atelectasia obstructiva o de una condensación mucopurulenta. Esta diferenciación no es posible en un estudio no contrastado ${ }^{1}$. Por su ubicación central, la mejor forma de observar y tomar muestras para biopsia de la lesión es una fibrobroncoscopía (FBC). Esta se ve beneficiada por el hecho, de que la mayoría de estos tumores tiende a situarse en zonas de bifurcación ${ }^{1,2}$. Un porcentaje importante de los tumores carcinoides $(30 \%)$ tienen algún grado de calcificación al momento de la FBC, incluso en algunos casos pueden tener una calcificación completa (osificación). Lo cual no sucedió con nuestra paciente, ya que el tumor a pesar de ser central, se encontraba friable, y con tejido de granulación.

La utilidad del PET-SCAN en el estudio de tumores carcinoides de origen pulmonar no es bien clara, debido a que tiene una sensibilidad que puede variar desde 14 a $100 \%$, dado a la escasa actividad que presenta con la fluorodesoxiglucosa. No obstante, gracias a que los tumores carcinoides contienen receptores de somatostatina, se ha comenzado a utilizar esta misma técnica, pero con análogos de la somatostatina, los cuales ayudan a la localización del tumor ${ }^{1}$. Dentro de los objetivos de la realización del PET-SCAN fue visualizar posibles lugares de metástasis. Sin embargo, este tipo de tumores, sobre todo el carcinoide típico, suelen tener escasa probabilidad de metastizar, aun así, existe un 3 a $5 \%$ de posibilidades de metástasis a distancia, las cuales son más frecuentes en hígado, huesos, glándula suprarrenal, y cerebro. En nuestra paciente, no se encontraron lesiones metastásicas, lo cual concuerda con el comportamiento típico de este tipo de carcinoides.

En el caso de nuestra paciente, además de la búsqueda de metástasis, el PET-SCAN era de gran utilidad para descartar algún otro origen del tumor carcinoide, debido a que este tipo de TNE es más usual de encontrar a nivel de tracto gastrointestinal $(90 \%)^{1}$, por lo cual, era necesario descartar la presencia de un tumor primario extrapulmonar.

En cuanto a la clínica, este tipo de tumores tienden a ser poco sintomáticos, sobre todo los de grado de malignidad bajo e intermedio, como lo son los tumores carcinoides. Es por ello, que no es de extrañar que la presentación clínica del cuadro en nuestra paciente haya sido tan inespecífico en un principio, simplemente con infecciones respiratorias bajas repetidas, que no produjeron extrañeza en los clínicos, hasta que la masa se hizo evidente en el estudio radiológico. A pesar, que la presentación de síndromes paraneoplásicos secundarios a la hipersecreción de ciertos compuestos neuroendocrinos, como la serotonina, y ACTH son característicos de este tipo de tumores, es de importancia recordar que lo son en su mayoría en los tumores de origen gastrointestinal, es por ello, que nuestra paciente nunca presentó este tipo de síndromes, como el síndrome carcinoide y de Cushing respectivamente ${ }^{1}$.

El tratamiento de elección en pacientes que presentan TNE de bajo grado de malignidad es el quirúrgico. Sin embargo, siempre el menos invasivo posible es una alternativa deseable. Dentro de estas técnicas mínimamente invasivas están la resección endobronquial o citorreducción mecánica con $\mathrm{FBC}$, láser, crioterapia, o electrocauterización $^{6,7}$. Este tipo de tumores, no son sensibles a la quimioterapia, por lo cual siempre la primera opción será la quirúrgica. Incluso en casos de metástasis, cuando son lesiones únicas, pueden ser removidas en el primer acto quirúrgico, como lo son las lesiones únicas hepáticas ${ }^{6}$. Las técnicas quirúrgicas son múltiples, siendo las más frecuentes la lobectomía y la resección limitada al tumor. Sin embargo, es la primera opción la que presenta una mejor sobrevida con 20 años en promedio, versus 15 años con la resección limitada.

Pacientes como la de nuestro caso clínico, con un TNE carcinoide típico no metastizado, que son sometidos a resección quirúrgica, presentan una sobrevida a 5 años de aproximadamente el 100\%, por lo cual, es menester aprovechar este buen pronóstico, para aplicar un tratamiento oportuno que minimice al máximo todo tipo de complicación a largo plazo 6 .

\section{Conclusiones y recomendaciones}

Los TNE como entidades neoplásicas, en general siguen siendo poco frecuentes, y sea cual sea su origen tienen una forma de presentación clínica, epidemiología, tratamiento y pronóstico muy distinto entre los diferentes tipos, y aún más según su origen embriológico. Como ya hemos visto los tumores de origen pulmonar constituyen la presentación menos frecuente, y dentro de estos, el tipo carcinoide típico es uno de los menos frecuentes, es por ello, que llama la atención el diagnóstico de nuestra paciente, además, por su forma de presentación clínica, ya que en este tipo de tumores tiende a ser un tanto confundente, e inespecífico.

Es necesario un alto nivel de sospecha por parte del clínico, no para el diagnóstico de un TNE, 
sino para saber diagnosticar a tiempo cuadros atípicos y de evolución tórpida, que nos orientan a cuadros poco frecuentes como estos. Sobre todo, con el avance tecnológico en la medicina, estas últimas décadas, han facilitado enormemente el diagnóstico de este tipo de entidades, ya que, el apoyo imageneológico ha sido de gran ayuda, además de los estudios mínimamente invasivos, que además permiten la toma de muestras que confirman el diagnóstico, e incluso la realización de tratamiento cuando es posible.

El aporte de este tipo de casos clínicos está dado principalmente para marcar precedente de entidades poco frecuentes, y orientar en el futuro estudio de pacientes con este tipo de patologías poco conocidas, debido a su escasa frecuencia, además de impulsar el avance en la mejora de los estudios a realizar en cada paciente, y según cada entidad.

\section{Bibliografía}

1.- DINCER H, PODGAETZ E, ANDRADE R. Pulmonary Neuroendocrine Tumors Part I. Spectrum and caracte- ristics of tumors. J Bronchol Intervent Pulmonol 2015; 22: $267-73$.

2.- SÁNCHEZ J. Diagnóstico y tratamiento de los tumores neuroendocrinos de pulmón. Arch. Bronconeumol 2014; 50: 392-6.

3.- GUSTAFSSON B, KIDD M, CHAN A, MALFERTHEINER M, MODLIN I. Bronchopulmonary Neuroendocrine Tumors. Amer. Cancer Society 2008; 113: 5-21.

4.- VILLA M, RONQUILlO C, MORÁN A, DIP A. Tumores Neuroendocrinos: experiencia de 6 años en un centro de tercer nivel. Gaceta Mexicana de Oncología 2015; 14: 141-9.

5.- BARBIERI F, ALBERTELLI M, GRILLO F, MOHAMED A, SAVEANU A, BARLIER A, et al. Neuroendocrine tumors: insights into innovative therapeutic options and rational development of targeted therapies. Drug Discovery Today 2014; 19: 458-68.

6.- DINCER H, PODGAETZ E, ANDRADE R. Pulmonary Neuroendocrine Tumors Part II. Treatment. J Bronchol Intervent Pulmonol 2015; 22: 351-6.

7.- QUINTERO J, ROJAS JC, SÁNCHEZ J, JARAMILLO L. Tumores neuroendocrinos del pulmón. Univ Med Bogotá 2008; 49: 413-23.

Correspondencia a:

Josefina Caviedes Morales

Interna de Medicina-Universidad San Sebastián,

Santiago.

Lota 2465, Providencia, Región Metropolitana.

Email: josefinacaviedes.m@gmail.com 